\title{
Multigrid method based on a space-time approach with standard coarsening for parabolic problems
}

\author{
Sebastião Romero Franco ${ }^{\mathrm{a}, \mathrm{b}}$, Francisco José Gasparc, Marcio Augusto Villela \\ Pinto $^{\mathrm{d}}$, Carmen Rodrigo ${ }^{\mathrm{e}, *}$ \\ a Department of Mathematics, State University of Centro-Oeste, Irati, PR, Brazil \\ ${ }^{\mathrm{b}}$ Graduate Program in Numerical Methods in Engineering, Federal University of Paraná, Curitiba, PR, Brazil \\ ${ }^{\text {c } C e n t r u m ~ W i s k u n d e ~ E ~ I n f o r m a t i c a ~(C W I), ~ S c i e n c e ~ P a r k ~ 123, ~ P .0 . ~ B o x ~ 94079, ~ A m s t e r d a m ~ 1090, ~ T h e ~ N e t h e r l a n d s ~}$ \\ d Department of Mechanical Engineering, Federal University of Paraná, Curitiba, PR, Brazil \\ e IUMA and Applied Mathematics Department, University of Zaragoza, María de Luna, 3, Zaragoza 50018, Spain
}

\section{A R T I C L E I N F O}

\section{MSC:}

00-01

99-00

Keywords:

Space-time multigrid

Local Fourier analysis

Parabolic partial differential equations

Double discretization

\begin{abstract}
A B S T R A C T
In this work, a space-time multigrid method which uses standard coarsening in both temporal and spatial domains and combines the use of different smoothers is proposed for the solution of the heat equation in one and two space dimensions. In particular, an adaptive smoothing strategy, based on the degree of anisotropy of the discrete operator on each grid-level, is the basis of the proposed multigrid algorithm. Local Fourier analysis is used for the selection of the crucial parameter defining such an adaptive smoothing approach. Central differences are used to discretize the spatial derivatives and both implicit Euler and Crank-Nicolson schemes are considered for approximating the time derivative. For the solution of the second-order scheme, we apply a double discretization approach within the space-time multigrid method. The good performance of the method is illustrated through several numerical experiments.
\end{abstract}

(c) 2017 Elsevier Inc. All rights reserved.

\section{Introduction}

We consider the heat equation $u_{t}-\Delta u=f$ in a bounded spatial domain $\Omega \subset \mathbb{R}^{d}$, with $d \in\{1,2\}$, on the bounded time interval $(0, T]$ as model problem. The central difference scheme on a regular mesh with grid-size $h$ is used for the spatial discretization, whereas the $\theta$-method is considered for the temporal approximation on a grid with constant spacing $\tau$. In particular, we will analyze the implicit Euler $(\theta=1)$ and the Crank-Nicolson $(\theta=1 / 2)$ schemes. The full space-time discretization results in a linear system of equations to solve, whose coefficient matrix depends on parameter

$$
\lambda=\tau / h^{2}
$$

\footnotetext{
* Corresponding author.

E-mail addresses: romero@unicentro.br (S.R. Franco), F.J.Gaspar@cwi.nl (F.J. Gaspar), marcio_villela@ufpr.br (M.A. Villela Pinto), carmenr@unizar.es (C. Rodrigo).
} 
which measures the degree of anisotropy in the discrete problem. In stencil notation the considered $\theta$-method for the one(space)dimensional model problem can be written as

$$
\left[\begin{array}{ccc}
0 & 0 & 0 \\
-\lambda \theta & 1+2 \lambda \theta & -\lambda \theta \\
-\lambda(1-\theta) & 2 \lambda(1-\theta)-1 & -\lambda(1-\theta)
\end{array}\right] .
$$

Notice that $\theta=1$ gives rise to the first order implicit Euler method, whereas the choice $\theta=1 / 2$ provides the stencil corresponding to the Crank-Nicolson approach.

Multigrid methods are among the most efficient techniques for solving systems of equations arising from the discretization of partial differential equations. Their success lies in their optimal complexity resulting from combining the smoothing property of standard iterative methods with the coarse-grid correction principle. Multigrid convergence strongly depends on the choice of the algorithm components. In particular, it is crucial to find a good interplay between the two main components: the smoothing method and the coarse-grid correction algorithm. For anisotropic problems, there are two strategies which give rise to good convergence rates. On the one hand, a standard smoothing procedure can be combined with a semicoarsening strategy doubling the mesh size in those directions in which the error is smooth. On the other hand, standard coarsening can be used if a stronger relaxation is considered. In particular, following Brandt's fundamental block relaxation rule [1], the unknowns which are strongly coupled must be simultaneously updated.

During the last decade, a new generation of supercomputers has appeared. Thus, to exploit such architectures, one must develop algorithms which are capable to efficiently use large number of cores. When solving time-dependent problems, the classic approach (time-stepping) is based on solving sequentially the problem for one time step after the other. However, this strategy does not allow the parallelization of the temporal variable, and in many practical problems very large number of time-steps are required. This led us to look for the increase of the concurrency by using time-parallel and full spacetime methods. There are different multigrid approaches on space-time grids, among which the space-time multigrid [2], the space-time concurrent multigrid waveform relaxation [3], the multigrid-reduction-in-time [4], the space-time parallel multigrid [5], the parallel full approximation scheme in space and time [6] and the parareal method [7,8] are included. In this work, also a multigrid method to deal with the entire space-time problem, opposite to the standard time-stepping strategy, is proposed for the solution of the heat equation with one and two spatial dimensions. The proposed approach is mainly based on a parameter-dependent choice of the smoother within the multigrid strategy.

Local Fourier analysis (LFA) or local mode analysis is a commonly used approach for analyzing the convergence properties of geometric multigrid methods. This analysis is based on the Fourier transform theory and requires the assumption of some hypotheses for its application. LFA was introduced by Brandt [9] and afterward extended in [10]. A good introduction can be found in the paper by Stüben and Trottenberg [11] and in the books by Wesseling [12], Trottenberg et al. [13], and Wienands and Joppich [14]. Local Fourier analysis is a useful tool in providing quantitative convergence estimates for multigrid algorithms. Such estimates can be rigorously justified in cases when the boundary conditions are periodic, but it is also known that LFA provides accurate predictions for the asymptotic convergence rates of multigrid methods for problems with other types of boundary conditions as well. In this work, we apply the local Fourier analysis to obtain the critical value $\lambda_{\text {crit }}$ which defines the adaptive smoothing strategy.

The rest of the paper is organized as follows. In Section 2 the space-time multigrid method for the heat equation with one (spatial) dimension is detailed, presenting also the local Fourier analysis that we use to define the adaptive smoothing strategy and the double discretization technique applied in the case of the Crank Nicolson scheme. After that, the extension of the proposed methodology is extended to the problem with two (spatial) dimensions in Section 3. The good performance of the presented algorithms is demonstrated in Section 4, where different numerical tests for one and two (spatial) dimensions are shown. Finally, conclusions are drawn in Section 5.

\section{Space-time multigrid method}

This section is focused on the introduction of the proposed space-time multigrid method and its analysis by means of local Fourier analysis. For ease of presentation, here we restrict ourselves to the one-dimensional heat equation.

\subsection{Space-time multigrid}

We describe the components of the proposed space-time multigrid method based on an adaptive smoothing strategy depending on the degree of anisotropy of the grids in the hierarchy. Parameter $\lambda$ defined in (1) indicates the degree of anisotropy in the discrete operator, which definitely has to be taken into account in the choice of the multigrid components. For large and small values of $\lambda$ the fully discrete problem is a strongly anisotropic problem in space and time respectively. Multigrid methods can deal with anisotropy in the operator by using different types of coarsening on the one hand, or special smoothers on the other hand. Oppositely to [2], where different coarsening strategies are used, we propose a space-time multigrid method which combines different smoothers coupling the strong connections in the stencil depending on parameter $\lambda$. Notice that the value of $\lambda$ varies from grid-level to grid-level in the hierarchy, and therefore convenient smoothers have to be chosen in the different meshes. 
Coarsening strategy. The proposed space-time multigrid method chooses a standard coarsening strategy to construct the grid-hierarchy, that is, the coarse-grid size doubles the fine-grid size in each direction (both temporal and spatial directions). Moreover, appropriate prolongation and restriction operators are chosen. In particular the prolongation operator, $I_{2 h}^{h}$, is chosen so that it does not transfer information backwards in time, whereas the restriction operator, $I_{h}^{2 h}$, is asymmetric and transferring no information forward in time. More concretely, these operators are given as follows (see [2]),

$$
\left.I_{2 h}^{h}=\frac{1}{2}\right]_{0}\left[\begin{array} { l l l } 
{ 1 } & { 2 } & { 1 } \\
{ 1 } & { 2 } & { 1 } \\
{ 0 } & { 0 } & { 0 }
\end{array} \left[_{2 h}^{h}, \quad I_{h}^{2 h}=\frac{1}{8}\left[\begin{array}{lll}
0 & 0 & 0 \\
1 & 2 & 1 \\
1 & 2 & 1
\end{array}\right]_{h}^{2 h} .\right.\right.
$$

Smoothing strategy. An adaptive smoothing strategy, which depends on the value of parameter $\lambda$, is the key ingredient of the proposed space-time multigrid method. This approach is based on the choice, on each grid in the hierarchy, of a smoother which is suitable for the current value of $\lambda$. In particular we are going to consider the following smoothers for the parabolic problem in one space dimension:

- zebra line-in-time relaxation: all unknowns located at the same spatial node but at different time-steps are simultaneously updated. Notice that this can be done efficiently since the system matrix results to be bi-diagonal. Moreover, the resulting lines in the temporal direction are visited in a zebra-wise manner, in the way that first the unknowns laying at the odd spatial nodes are relaxed and after that we update those in the even spatial points.

- zebra line-in-space relaxation: it is the same algorithm as above but this time the lines are in the spatial direction and, of course, all unknowns at each line have to be updated simultaneously by solving the corresponding system of equations.

We propose a critical value $\lambda_{\text {crit }}$ which is used as a switch for choosing the smoothing procedure at any grid-level:

The value of $\lambda_{\text {crit }}$ will be obtained from the local Fourier analysis results presented in next section.

\subsection{Local Fourier analysis for the choice of $\lambda_{\text {crit }}$}

A key point for the good performance of the proposed space-time multigrid method is the choice of parameter $\lambda_{\text {crit }}$, indicating when to change from one smoother to another. This parameter is selected with the help of the local Fourier analysis, which is a quantitative analysis to study the properties of the smoother and the two-grid operator within the multigrid framework.

The basis of LFA is to formally extend all multigrid components to an infinite grid $\Omega_{h, \tau}$, neglecting the boundary conditions, and analyze discrete linear operators with constant coefficients. In this way, the eigenfunctions of such operators are some complex exponential functions called Fourier components, $\varphi_{h, \tau}(\boldsymbol{\theta}, x, t)=\mathrm{e}^{i \theta_{1} x} \mathrm{e}^{i \theta_{2} t}$, with $(x, t) \in \Omega_{h, \tau}$, and $\boldsymbol{\theta}=\left(\theta_{1}, \theta_{2}\right) \in \boldsymbol{\Theta}_{h, \tau}=(-\pi / h, \pi / h] \times(-\pi / \tau, \pi / \tau]$. These grid functions form a unitary basis for the space of bounded functions on the infinite grid, and define the Fourier space $\mathcal{F}\left(\Omega_{h, \tau}\right):=\operatorname{span}\left\{\varphi_{h, \tau}(\boldsymbol{\theta}, \cdot) \mid \boldsymbol{\theta} \in \boldsymbol{\Theta}_{h, \tau}\right\}$. If we assume that the error is a linear combination of the Fourier components, the behavior of the multigrid method can be analyzed by evaluating the error reduction associated with each particular multigrid component on the Fourier modes.

In order to investigate the interplay between relaxation and coarse-grid correction, it is necessary to perform at least a two-grid analysis. One needs to consider the error propagation operator from the two-grid method, $M_{h}^{2 h}=S_{h}^{\nu_{2}}\left(I_{h}-\right.$ $\left.I_{2 h}^{h} L_{2 h}^{-1} L_{h}^{2 h} L_{h}\right) S_{h}^{\nu_{1}}$, where $S_{h}$ is the smoothing procedure and the coarse-grid correction operator is composed of the discrete operators on the fine and coarse grids, $L_{h}$ and $L_{2 h}$, respectively, and the inter-grid transfer operators: restriction, $I_{h}^{2 h}$ and prolongation $I_{2 h}^{h}$. The two-grid analysis is the basis for the classical asymptotic multigrid convergence estimates, and the spectral radius of the two-grid operator, $\rho\left(M_{h}^{2 h}\right)$, indicates the asymptotic convergence factor of the two-grid method. To estimate this value, the crucial observation is that the coarse-grid correction operator, as well as the smoother, leave the so-called spaces of $2 h$-harmonics, $\mathcal{F}^{4}\left(\boldsymbol{\theta}^{00}\right)=\operatorname{span}\left\{\varphi_{h, \tau}\left(\boldsymbol{\theta}^{\alpha_{1} \alpha_{2}}, x, t\right) \mid \alpha_{1}, \alpha_{2} \in\{0,1\}\right\}$, with $\boldsymbol{\theta}^{00} \in \boldsymbol{\Theta}_{\text {low }}=(-\pi / 2 h, \pi / 2 h] \times$ $(-\pi / 2 \tau, \pi / 2 \tau]$, and $\boldsymbol{\theta}^{\alpha_{1} \alpha_{2}}=\boldsymbol{\theta}^{00}-\left(\alpha_{1} \operatorname{sign}\left(\theta_{1}^{00}\right) \pi, \alpha_{2} \operatorname{sign}\left(\theta_{2}^{00}\right) \pi\right)$, invariant. Then, $M_{h}^{2 h}$ is equivalent to a block-diagonal matrix consisting of blocks denoted by $\widetilde{M}_{h}^{2 h}\left(\theta^{00}\right)=\left.M_{h}^{2 h}\right|_{\mathcal{F}^{4}\left(\boldsymbol{\theta}^{00}\right)}$, that is, its Fourier domain representation. In this way, one can determine the spectral radius $\rho\left(M_{h}^{2 h}\right)$ by calculating the spectral radius of these smaller matrices, that is:

$$
\rho=\rho\left(M_{h}^{2 h}\right)=\sup _{\boldsymbol{\theta}^{00} \in \boldsymbol{\Theta}_{\text {low }}} \rho\left(\tilde{M}_{h}^{2 h}\left(\boldsymbol{\theta}^{00}\right)\right) .
$$

We propose to combine the use of two line-wise smoothers in alternate directions, one in the temporal direction and another in the spatial direction, both of them performed in a zebra manner. We analyze for which values of parameter $\lambda$ these relaxations provide good convergence for the multigrid algorithm. In Fig. 1 we show the two-grid convergence factors provided by the LFA, corresponding to the two multigrid methods based on the two considered smoothers, for different values of $\log _{2} \lambda$ ranging from -8 to 8 . We can observe that if the zebra-in-time smoother is used we obtain good results for small values of $\lambda$, whereas the zebra-in-space relaxation is suitable when $\lambda$ is large. This behavior can be well understood by analyzing the coefficients of the problem. For large or small values of $\lambda$ we are dealing with a highly anisotropic fully discrete problem. It is well known that the smoother must be performed in the direction of the strong connection of the unknowns if standard coarsening is used. Therefore, line-in-space relaxation will work well when there is strong coupling 


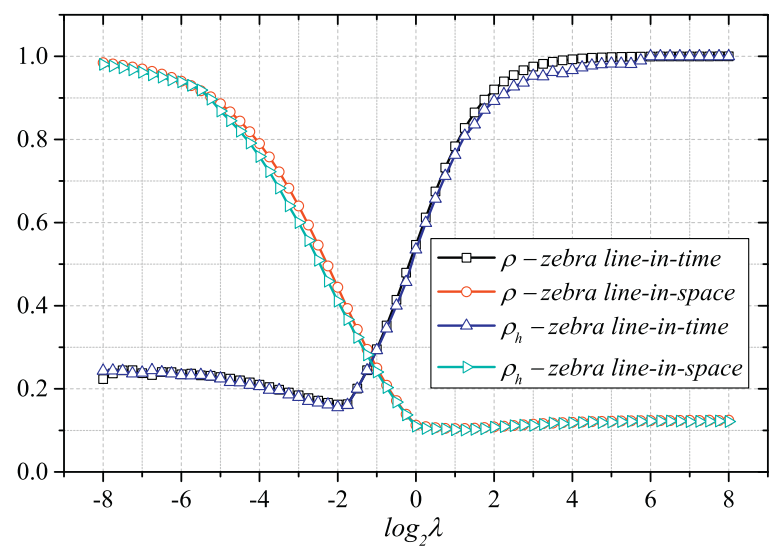

Fig. 1. Two-grid convergence factors predicted by LFA $(\rho)$ corresponding to multigrid based on zebra line-in-time and zebra line-in-space, and experimentally convergence factors $\left(\rho_{h}\right)$, for different values of parameter $\lambda$, for the one-(space)dimensional model problem.

along the spatial direction (large $\lambda$ ) whereas line-in-time will perform well in the case of strong connection in time (small $\lambda$ ). In order to confirm the predictions of the LFA, we also display the experimentally obtained convergence factors. In particular, these convergence rates are obtained as an average after 30 multigrid iterations.

We calculate that the value of $\lambda$ for which the convergence of the multigrid is the same by using both smoothers is $\lambda=0.46$, which therefore is chosen as the switch value $\lambda_{\text {crit }}$. This parameter will be considered in the numerical tests in Section 4.1 .

\subsection{Space-time multigrid for a second-order method}

The second order accurate Crank-Nicolson method is often used for parabolic problems as it reduces the total volume of calculation, being unconditionally stable, and only involves one stage per time step. Whereas the multigrid waveform relaxation provides satisfactory results with the Crank-Nicolson scheme [15], difficulties appear with methods using timecoarsening. For instance, in [2], the authors show that the space-time multigrid algorithm with standard coarsening in space and in time is nowhere optimal, performing very badly except for values of $\lambda$ close to 1 . In [16] Wu shows that parareal algorithms are not robust with respect to the discretization parameters if the Crank-Nicolson scheme is chosen. An interesting approach to overcome this difficulty in the context of parareal methods can be found in the recent work by the same author [17].

We propose here a space-time multigrid method for the Crank-Nicolson second-order scheme. This approach again is based on an adaptive strategy using a value $\lambda_{\text {crit }}$. This parameter is the changing point from which a zebra line-in-time based multigrid deteriorates for the Crank-Nicolson scheme. Therefore, we propose an algorithm in which if $\lambda \leq \lambda_{\text {crit }}$ the same zebra line-in-time smoother previously proposed for the Euler discretization is used in the space-time multigrid method for the Crank-Nicolson scheme. If $\lambda>\lambda_{\text {crit }}$ then the previous method does not work well anymore and we need to use a more sophisticated approach.

The multigrid technique offers possibilities to improve not only the algebraic convergence but also the accuracy of the discrete solution with respect to the analytical solution. This is the basic idea of double discretization, which can be seen as a special defect correction technique. We propose the use of the double-discretization approach proposed by Brandt [1]. It consists of employing a stable discretization scheme in the relaxation sweeps, which can be of lower order than desired, combined with the use of a discretization of the requested order to compute the transferred residuals. This strategy has already been successfully applied in several problems as fourth and sixth order approximations to Poisson equation [18], second-order approximations to singular perturbation problems [9,19] and in fluid dynamics [1,20]. In our case, the first order Euler scheme is used to do the relaxations on all levels, whereas the second order Crank-Nicolson scheme is used in calculating the residuals. This latter scheme determines the accuracy of the numerical solution.

From the above, our recommendation is to apply the space-time multigrid method with zebra line-in-time smoother for the Crank-Nicolson scheme when $\lambda \leq \lambda_{\text {crit }}$ on the finest grid, and otherwise, to perform a double discretization technique combined with the method proposed for the first-order scheme as shown in Algorithm 2. We want to remark that

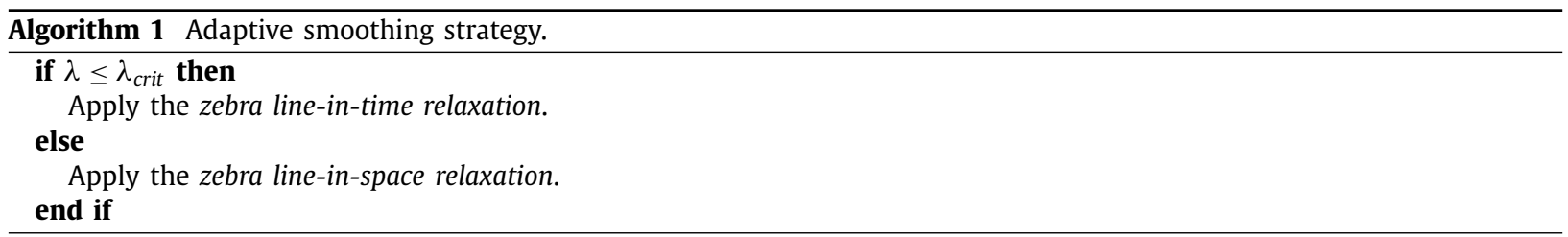




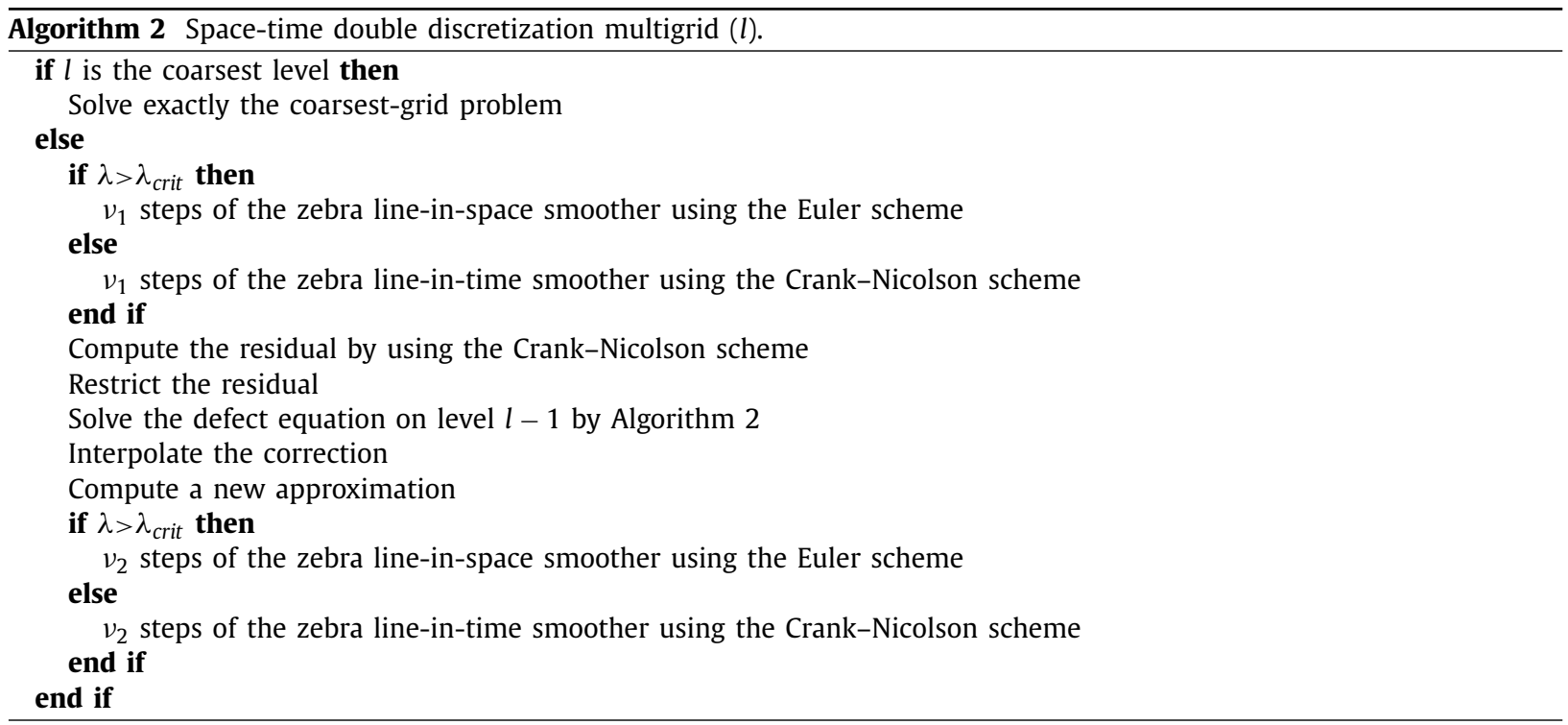

Algorithm 2 is only used when $\lambda>\lambda_{\text {crit }}$ on the finest grid.

\section{Extension to parabolic problems in two space dimensions}

In this section, we consider the extension of the proposed solution strategy to the case of parabolic problems in two space dimensions. The discrete operator corresponding to the $\theta$-method can be written in stencil form as follows:

$$
L_{h}=\left[\left[\begin{array}{ccc}
0 & -\lambda(1-\theta) & 0 \\
-\lambda(1-\theta) & 4 \lambda(1-\theta)-1 & -\lambda(1-\theta) \\
0 & -\lambda(1-\theta) & 0
\end{array}\right]\left[\begin{array}{ccc}
0 & -\lambda \theta & 0 \\
-\lambda \theta & 1+4 \lambda \theta & -\lambda \theta \\
0 & -\lambda \theta & 0
\end{array}\right]\left[\begin{array}{lll}
0 & 0 & 0 \\
0 & 0 & 0 \\
0 & 0 & 0
\end{array}\right]\right] .
$$

Regarding the extension of the space-time multigrid algorithm, the main difference falls on the smoothing part of the algorithm. Although the idea is the same as in the one-dimensional spatial case, we consider now line and plane smoothers. More concretely, the following relaxation methods are used within the algorithm:

- red-black line-in-time relaxation: it is similar to the zebra line-in-time relaxation previously described in the onedimensional case, with the only difference that the lines are visited in a red-black ordering within the two-dimensional spatial grid.

- zebra plane-in-space relaxation: all unknowns located at the same time-level are simultaneously updated, and the resulting planes are visited by following a zebra-wise manner. Theoretically, this smoother seems to be expensive due to the necessity of exactly solving the 2D problems arising in plane relaxation. However, in practice, usually it is not necessary to solve them exactly, and it is sufficient to apply one multigrid cycle within each plane (see $[1,13,21]$ for example). Here, we use only one $V$-cycle with one pre- and two post-smoothing steps for the plane relaxation, since this multigrid cycle results in a very efficient space-time multigrid algorithm.

Again, $\lambda_{\text {crit }}$ will be used for choosing the smoother. In this case, the red-black line-in-time relaxation will be considered if $\lambda \leq \lambda_{\text {crit }}$, whereas the zebra plane-in-space relaxation will be used otherwise.

The coarse-grid correction part of the algorithm is straightforwardly extended to two space dimensions, resulting the transfer grid operators as follows (see [2]),

$$
\begin{aligned}
& I_{2 h}^{h}=\frac{1}{4}\left[\begin{array}{lll}
0 & 0 & 0 \\
0 & 0 & 0 \\
0 & 0 & 0
\end{array}\left[\begin{array}{lll}
h \\
2 h
\end{array}\right] \begin{array}{lll}
1 & 2 & 1 \\
2 & 4 & 2 \\
1 & 2 & 1
\end{array}\left[\begin{array}{lll}
h \\
2 h
\end{array}\right] \begin{array}{lll}
1 & 2 & 1 \\
2 & 4 & 2 \\
1 & 2 & 1
\end{array}\left[\begin{array}{l}
h \\
2 h
\end{array}\right],\right. \\
& I_{h}^{2 h}=\frac{1}{32}\left[\left[\begin{array}{lll}
1 & 2 & 1 \\
2 & 4 & 2 \\
1 & 2 & 1
\end{array}\right]_{h}^{2 h}\left[\begin{array}{lll}
1 & 2 & 1 \\
2 & 4 & 2 \\
1 & 2 & 1
\end{array}\right]_{h}^{2 h}\left[\begin{array}{lll}
0 & 0 & 0 \\
0 & 0 & 0 \\
0 & 0 & 0
\end{array}\right]_{h}^{2 h}\right] .
\end{aligned}
$$

Notice that the previous stencil notation corresponds to a sequence of stencils applied to successive time-steps from the lowest (left stencil) to the highest (right stencil). 


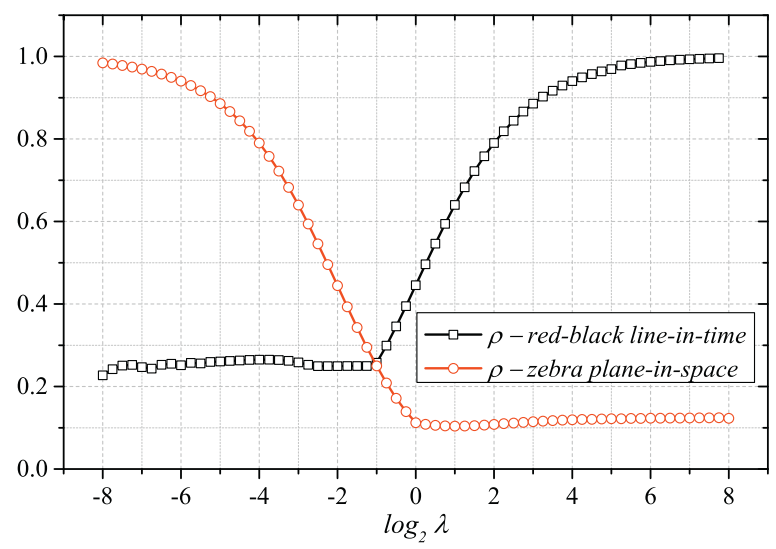

Fig. 2. Two-grid convergence factors predicted by LFA $(\rho)$ corresponding to multigrid based on zebra line-in-time and zebra line-in-space for different values of parameter $\lambda$, for the two-(space)dimensional model problem.

Remark 1. We wish to emphasize that the components of the proposed multigrid algorithm are not new. Their clever combination is however the key for a successful performance of the method. In fact, the restriction and prolongation operators are identical to the one used in the space-time multigrid method by Horton and Vandewalle [2]. The zebra line-intime smoother is the same smoother as the one used in the multigrid waveform relaxation [15]. Finally, the plane-in-space smoother is very similar to that used in the space-time parallel multigrid algorithm by Gander and Neumüller [5]. Whereas in the latter, a Jacobi variant is used, here a zebra-plane Gauss-Seidel is chosen. In both approaches, the exact solve is substituted by a single multigrid cycle with very satisfactory results.

Local Fourier analysis can be also implemented to provide insight in the case of two space dimensions. It results in a three-dimensional local Fourier analysis, taking into account the two spatial dimensions and the temporal one. Next, we present some results of such analysis to support the choice of $\lambda_{\text {crit }}$. Note that when analyzing the zebra plane-in-space relaxation we consider the exact solution of the whole system corresponding to all unknowns located in it. In practice, although we only apply a multigrid cycle to obtain an approximation of the solution, not solving it exactly, the convergence factors provided by the analysis are in a very good agreement with those experimentally obtained. We perform the same experiment than in the one-dimensional case, and in Fig. 2 we depict the two-grid convergence factors provided by LFA for different values of $\lambda$. In this case, the multigrid methods analyzed are those using the red-black line-in-time smoother and the zebra plane-in-space relaxation. Similarly to the previous case, the line-in-time smoother works successfully for small values of $\lambda$ but the plane-in-space relaxation is necessary when $\lambda$ becomes larger. This behavior is well understood using similar arguments as discussed earlier. We obtain the same value of $\lambda_{\text {crit }}$ as in the one-(space) dimensional case.

\section{Numerical results}

In this section, we present some numerical experiments in order to demonstrate the efficiency and good performance of the space-time multigrid method proposed in previous sections. First, we consider the one-(space)dimensional model problem in order to explain the choice of the remaining details of the algorithm. We test the behavior of the space-time multigrid method for both the first- and second-order approaches. After that, we extend the suggested strategy to the two-(space)dimensional heat equation, including the double discretization technique for the solution of the Crank-Nicolson scheme.

\subsection{One-dimensional heat equation}

First, we demonstrate the good behavior of the space-time multigrid method proposed for the heat equation in the one-spatial dimension domain $\Omega=(0,1)$. The right-hand side and the boundary conditions are chosen such that the exact solution is given by $u(x, t)=e^{-t} \sin (\pi x)$.

\section{First-order Euler scheme}

We consider an Euler scheme for the discretization in time of the model problem, that is, the discrete operator is given by (2) with $\theta=1$. Next, we choose the type of cycle which will be used in the rest of the experiments and we show the robustness of this selection.

First of all, we would like to test the use of $V$ - and F-cycles within the algorithm proposed in Section 2.1. For this purpose, in Fig. 3(a) we show the convergence rates provided by both cycle approaches, considering only one post-smoothing step, for different values of $\lambda$. The value of $\lambda_{\text {crit }}$ is chosen as 0.46 following the suggestion given by the predictions of LFA 

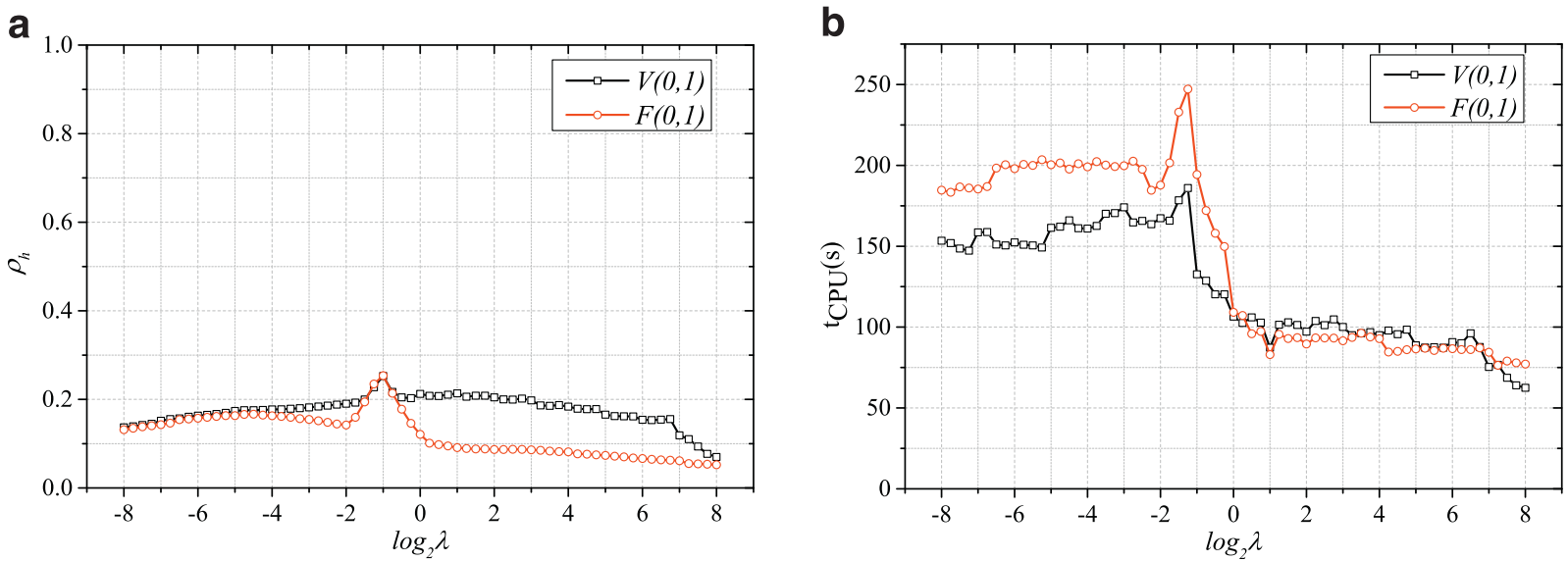

Fig. 3. (a) Convergence rates and (b) CPU times for the proposed space-time multigrid method based on $V$ - and $F$-cycles with one post-smoothing step, for a wide range of values of parameter $\lambda$.

Table 1

Number of iterations needed to reduce the maximum norm of the initial residual by a factor of $10^{-10}$, for different values of parameter $\lambda$ and different grid-sizes $\left(n_{x} \times n_{t}\right)$ with fixed $h=1 / n_{x}$ and increasing number of time-steps $n_{t}$.

\begin{tabular}{llllllll}
\hline \multicolumn{8}{c}{$\log _{2} \lambda$} \\
\hline$n_{x} \times n_{t}$ & $2^{10} \times 2^{10}$ & $2^{10} \times 2^{11}$ & $2^{10} \times 2^{12}$ & $2^{10} \times 2^{13}$ & $2^{10} \times 2^{14}$ & $2^{10} \times 2^{15}$ & $2^{10} \times 2^{16}$ \\
\hline-8.0 & 11 & 12 & 12 & 12 & 12 & 12 & 12 \\
-6.0 & 13 & 13 & 13 & 13 & 13 & 13 & 13 \\
-4.0 & 13 & 13 & 13 & 13 & 14 & 14 & 14 \\
-2.0 & 14 & 14 & 14 & 14 & 14 & 14 & 14 \\
0.0 & 15 & 15 & 15 & 15 & 15 & 15 & 15 \\
2.0 & 15 & 15 & 15 & 15 & 15 & 15 & 15 \\
4.0 & 13 & 14 & 14 & 14 & 14 & 14 & 14 \\
6.0 & 11 & 12 & 13 & 13 & 13 & 13 & 13 \\
8.0 & 8 & 9 & 9 & 9 & 9 & 9 & 9 \\
\hline
\end{tabular}

Table 2

Maximum norm of the error, $\|e\|_{\infty}$, obtained on each iteration (it) until the discretization error is achieved.

\begin{tabular}{llllllllll}
\hline it & 3 & 4 & 5 & 6 & 7 & 8 & 9 & 10 & 11 \\
\hline$\|e\|_{\infty}$ & $5.1 \mathrm{E}-3$ & $7.9 \mathrm{E}-4$ & $1.4 \mathrm{E}-4$ & $2.9 \mathrm{E}-5$ & $7.7 \mathrm{E}-6$ & $5.3 \mathrm{E}-6$ & $5.6 \mathrm{E}-6$ & $5.7 \mathrm{E}-6$ & $5.7 \mathrm{E}-6$ \\
\hline
\end{tabular}

in Section 2.2. The range of parameter $\lambda$, denoting the anisotropy of the discrete operator on the finest grid, is given by $-8 \leq \log _{2} \lambda \leq 8$, sampled in increments of 0.25 . We observe that both multigrid cycles provide very satisfactory convergence factors for all values of $\lambda$. In Fig. 3(b), we compare the CPU time needed to reduce the maximum norm of the initial residual by a factor of $10^{-10}$ by using both approaches based on $V$ - and F-cycles. Looking at the whole interval of values of $\lambda$, it seems that the computational cost of both cycles is comparable. For this reason, together with the fact that straightforward parallelization is optimal for $V$-cycles, opposite to $W$ - and $F$-cycles which scale poorly, in the following we choose to use $V$-cycle within the proposed space-time multigrid algorithm.

Next, we show the robustness of the method based on $V(0,1)$-cycles with respect of the grid-size. In Table 1 , we display the number of cycles needed to reduce the maximum norm of the initial residual by a factor of $10^{-10}$ for different values of $\lambda$ and for different meshes. In particular, we fix the number of spatial steps $n_{x}=2^{10}$ and we consider an increasing number of time-steps $n_{t}$ varying from $2^{10}$ to $2^{16}$. We can observe a very satisfactory grid-independent convergence of the method that makes the use of the proposed space-time multigrid algorithm very attractive.

\section{Second-order approach}

Next, we study the performance of the algorithm presented in Section 2.3 for the second-order discretization of our model problem, that is (2) with $\theta=1 / 2$. It is well-known that such a multigrid method does not converge to zero residuals, so it is necessary to directly check the convergence to the differential solution. Thus, in Table 2 we show the maximum norm of the error for the different multigrid iterations by using $\lambda=2^{4}$ as an example. A grid with $h=\tau=2^{-8}$ has been considered, and we can observe that after only ten iterations the discretization error is already achieved, which makes this method very appealing. 
Table 3

Maximum norm of the error, $\|e\|_{\infty}$, for different grid-sizes given by $\Delta x=1 / n_{x}$ and $\Delta t=1 / n_{t}$, with $n_{x}=n_{t}$ varying from $2^{6}$ to $2^{13}$.

\begin{tabular}{lllllllll}
\hline$n_{x}=n_{t}$ & $2^{6}$ & $2^{7}$ & $2^{8}$ & $2^{9}$ & $2^{10}$ & $2^{11}$ & $2^{12}$ & $2^{13}$ \\
\hline$\|e\|_{\infty}$ & $3.4 \mathrm{E}-4$ & $9.1 \mathrm{E}-5$ & $2.4 \mathrm{E}-5$ & $6.0 \mathrm{E}-6$ & $1.5 \mathrm{E}-6$ & $3.8 \mathrm{E}-7$ & $9.6 \mathrm{E}-8$ & $2.4 \mathrm{E}-8$ \\
\hline
\end{tabular}

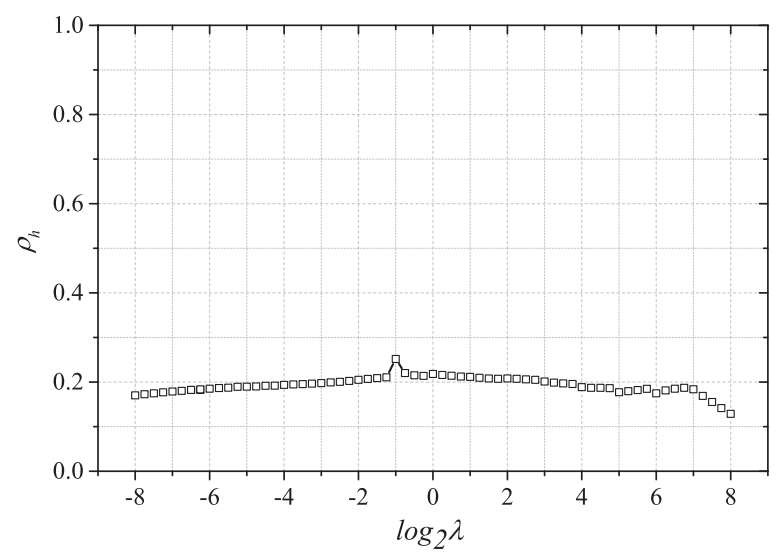

Fig. 4. Convergence rates for the proposed space-time multigrid method based on double discretization technique for the one-dimensional problem, for a wide range of values of parameter $\lambda$.

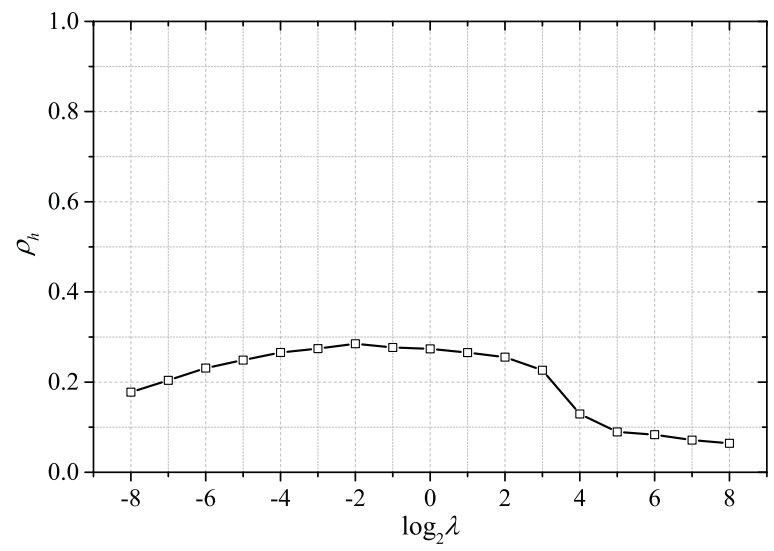

Fig. 5. Convergence rates for the proposed space-time multigrid method for the two-dimensional problem, for a wide range of values of parameter $\lambda$.

In order to ensure that the double discretization technique provide us the desired second-order accuracy, in Table 3 we display the maximum norm of the error for different grid-sizes varying from $h=2^{-6}$ to $h=2^{-13}$ and with $h=\tau$. We can see that when the grid-sizes are halved the errors are reduced by one-fourth, which implies the second-order accuracy of the Crank-Nicolson scheme.

Finally, to show the good performance of the proposed method, in Fig. 4 we display the convergence factors $\rho_{h}$ obtained for a wide range of values of $\lambda$, after 20 iterations. We can observe that for any value of parameter $\lambda$ the convergence rates are between 0.1 and 0.25 , providing very satisfactory results which are almost identical to the convergence showed in Fig. 3 from the space-time method for the first-order Euler scheme.

\subsection{Two-dimensional heat equation}

In this section, we consider the two-(space)dimensional heat equation in the square domain $\Omega=(0,1) \times(0,1)$. The right-hand side and the boundary conditions are chosen such that the exact solution of the problem is $u(x, y, t)=$ $e^{-t} \sin \pi x \sin \pi y$. 
Table 4

Number of iterations needed to reduce the maximum norm of the initial residual by a factor of $10^{-10}$, for different values of parameter $\lambda$ and different grid-sizes $\left(n_{x} \times n_{y} \times n_{t}\right)$ with fixed $h=1 / n_{x}=1 / n_{y}=2^{-7}$ and increasing number of time-steps $n_{t}$

\begin{tabular}{lllllll}
\hline \multicolumn{7}{c}{$\log _{2} \lambda$} \\
\hline$n_{t}$ & $2^{7}$ & $2^{8}$ & $2^{9}$ & $2^{10}$ & $2^{11}$ & $2^{12}$ \\
\hline-8 & 10 & 10 & 11 & 12 & 13 & 14 \\
-6 & 12 & 13 & 14 & 15 & 15 & 16 \\
-4 & 15 & 16 & 16 & 17 & 17 & 18 \\
-2 & 18 & 18 & 18 & 19 & 19 & 19 \\
0 & 18 & 18 & 18 & 18 & 18 & 18 \\
2 & 13 & 17 & 17 & 17 & 17 & 17 \\
4 & 10 & 10 & 12 & 12 & 12 & 12 \\
6 & 9 & 10 & 10 & 10 & 10 & 10 \\
8 & 9 & 9 & 9 & 9 & 9 & 9 \\
\hline
\end{tabular}

Table 5

Maximum norm of the error, $\|e\|_{\infty}$, for different grid-sizes, given by $h=1 / n_{x}=$ $1 / n_{y}$ and $\tau=1 / n_{t}$, with $n_{x}=n_{y}=n_{t}$ varying from $2^{3}$ to $2^{8}$.

\begin{tabular}{lllllll}
\hline$n_{x}=n_{y}=n_{t}$ & $2^{3}$ & $2^{4}$ & $2^{5}$ & $2^{6}$ & $2^{7}$ & $2^{8}$ \\
\hline$\|e\|_{\infty}$ & $1.2 \mathrm{E}-2$ & $3.6 \mathrm{E}-3$ & $1.1 \mathrm{E}-3$ & $3.4 \mathrm{E}-4$ & $9.6 \mathrm{E}-5$ & $2.6 \mathrm{E}-5$ \\
\hline
\end{tabular}

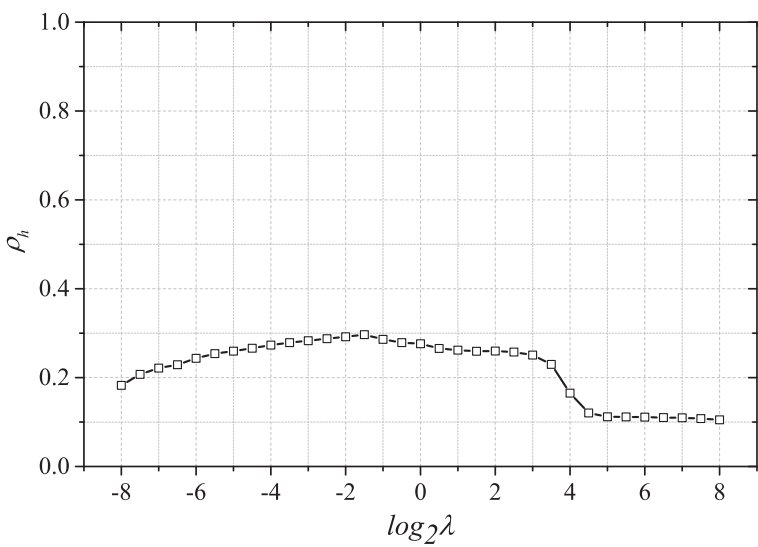

Fig. 6. Convergence rates for the proposed space-time multigrid method based on double discretization technique for the two-dimensional problem, for a wide range of values of parameter $\lambda$.

\section{First-order Euler scheme}

First, we consider the discrete problem obtained by using central differences for the spatial derivatives and the implicit Euler scheme for the temporal discretization. We apply the extension of the proposed space-time multigrid method to the case of two-(space)dimensions. Therefore, as selected in the previous numerical experiment, a $V$-cycle with only one postsmoothing step is considered to run the simulations.

In Fig. 5 we show the convergence factors provided by such algorithm for different values of parameter $\lambda$. The stopping criterion to obtain these convergence rates is to reduce the maximum norm of the initial residual in a factor of $10^{-10}$. We can observe a very satisfactory convergence for all values of the parameter $\lambda$. For all cases, the obtained rates are below 0.3 .

Next, in Table 4, we display the number of multigrid iterations necessary to reduce the maximum norm of the initial residual by a factor of $10^{-10}$. The results are shown for different values of parameter $\lambda$ ranging from -8 to 8 , and for different grid-sizes. The number of spatial steps are fixed as $n_{x}=n_{y}=2^{7}$, whereas different numbers of time-steps are considered $2^{7}<n_{t}<2^{12}$. We can observe the robustness of the proposed method, which provides very satisfactory results for the different values of $\lambda$ considered, being particularly efficient for large values of this parameter.

\section{Second order approach}

Next, we analyze the behavior of the extension of Algorithm 2 to two space dimensions. As in the one-(space)dimensional case, we have observed that the convergence to the differential solution is satisfactorily achieved after only 11 iterations, 
when a finest grid with $h=2^{-8}$ and $\log _{2} \lambda=4$ are considered. Also, as shown in Table 5 , we ensure that the double discretization technique provides us the desired second-order accuracy. In such Table 5 we display the reduction of the maximum norm of the error for different grid-sizes varying from $h=2^{-3}$ to $h=2^{-8}$ and with $h=\tau$. As expected, the errors are reduced by one-fourth when the grid-sizes are halved, demonstrating the second-order convergence determined by the Crank-Nicolson scheme.

To conclude the good behavior of the proposed method is displayed in Fig. 6, where the convergence rates $\rho_{h}$ obtained for a wide range of values of $\lambda$, after 20 iterations are shown. For any value of parameter $\lambda$ the convergence of the method results very satisfactory, giving rise to convergence rates between 0.1 and 0.3 . These results are very similar to those showed for the first-order Euler scheme in Fig. 5.

\section{Conclusions}

In this work, a robust space-time multigrid method combining standard coarsening with the selection of suitable smoothers depending on the anisotropy of the problem has been presented for solving the heat equation. The choice of the smoother is done by using a critical value of parameter $\lambda$, which represents the degree of anisotropy in the discrete operator, resulting in an adaptive smoothing strategy. This parameter has been selected with the help of a local Fourier analysis. One and two space dimensions are considered in the study and the temporal approximation of the problem is performed by using the well-known first- and second-order schemes corresponding to Euler and Crank Nicolson methods. This latter is solved by applying a double discretization technique within the space-time multigrid algorithm. This is one of the strengths of the proposed method, since the literature fails to provide a space-time multigrid method with time-coarsening for the Crank-Nicolson scheme. For all test problems, very good convergence rates are provided for a wide range of values of parameter $\lambda$. Further work will include the extension of the proposed space-time multigrid method to more complicated problems, in particular in the field of porous media.

\section{References}

[1] A. Brandt, O. Livne, Multigrid Techniques: 1984 Guide with Applications to Fluid Dynamics, Society for Industrial and Applied Mathematics, 2011, doi:10.1137/1.9781611970753.

[2] G. Horton, S. Vandewalle, A space-time multigrid method for parabolic partial differential equations, SIAM J. Sci. Comput. 16 (4) (1995) 848-864, doi:10.1137/0916050.

[3] G. Horton, S. Vandewalle, P. Worley, An algorithm with polylog parallel complexity for solving parabolic partial differential equations, SIAM J. Sci. Comput. 16 (3) (1995) 531-541.

[4] R.D. Falgout, S. Friedhoff, T.V. Kolev, S.P. MacLachlan, J.B. Schroder, Parallel time integration with multigrid, SIAM J. Sci. Comput. 36 (6) (2014) C635-C661.

[5] M.J. Gander, M. Neumüller, Analysis of a new space-time parallel multigrid algorithm for parabolic problems, SIAM J. Sci. Comput. 38 (4) (2016) A2173-A2208, doi:10.1137/15M1046605.

[6] M. Emmett, M.L. Minion, Toward an efficient parallel in time method for partial differential equations, Commun. Appl. Math. Comput. Sci. 7 (2012) $105-132$.

[7] J.-L. Lions, Y. Maday, G. Turinici, A “parareal” in time discretization of PDE's, C. R. Acad. Sci. Ser. I Math. 332 (2001) 661-668.

[8] M.J. Gander, S. Vandewalle, Analysis of the parareal time-parallel time-integration method, SIAM J. Sci. Comput. 29 (2) (2007) 556-578.

[9] A. Brandt, Multi-level adaptive solutions to boundary-value problems, Math. Comput. 31 (138) (1977) 333-390.

[10] A. Brandt, Rigorous quantitative analysis of multigrid: I. Constant coefficients two-level cycle with $L_{2}$-norm, SIAM J. Numer. Anal. 31 (6) (1994) $1695-1730$.

[11] K. Stüben, U. Trottenberg, Multigrid methods: fundamental algorithms, model problem analysis and applications, in: W. Hackbusch, U. Trottenberg (Eds.), Multigrid Methods, Lecture Notes in Mathematics, 960, Springer Berlin Heidelberg, 1982, pp. 1-176.

[12] P. Wesseling, An Introduction to Multigrid Methods, John Wiley, Chichester, UK, 1992.

[13] U. Trottenberg, C.W. Oosterlee, A. Schüller, Multigrid, Academic Press, New York, 2001.

[14] R. Wienands, W. Joppich, Practical Fourier Analysis for Multigrid Methods, Chapman and Hall/CRC Press, 2005.

[15] S. Vandewalle, G. Horton, Fourier mode analysis of the multigrid waveform relaxation and time-parallel multigrid methods, Computing 54 (4) (1995) 317-330, doi:10.1007/BF02238230.

[16] S. Wu, Convergence analysis of some second-order parareal algorithms, IMA J. Numer. Anal. 35 (3) (2015) 1315-1341, doi:10.1093/imanum/dru031.

[17] S. Wu, T. Zhou, Convergence analysis for three parareal solvers, SIAM J. Sci. Comput. 37 (2) (2015) A970-A992, doi:10.1137/140970756.

[18] S. Schaeffer, High-Order Multigrid Methods. Ph.D. thesis, Colorado State University, Fort Collins, Colorado, 1982.

[19] C. Börgers, Mehrgitterverfahren für eiene Mehrstellendiskreitsierung der Poissongleichung und für eiene zweidimensionale singulär gestörte Aufgabe. Ph.D. thesis, Institut für Angewandte Mathematik, Universität Bonn, 1981.

[20] R. Jyotsna, S.P. Vanka, A pressure based multigrid procedure for the Navier-Stokes equations on unstructured grids, in: N.D. Melson, T.A. Manteuffel, S.F. Mc.Cormick, C.C. Douglas (Eds.), Seventh Copper Mountain Conference on Multigrid Methods NASA Conference Publication, 3339, NASA Hampton, 1996, pp. 409-424.

[21] I.M. Llorente, N.D. Melson, Robust multigrid smoothers for three dimensional elliptic equations with strong anisotropies, ICASE, 1998. Technical report 98-37. 